\title{
A study of reflection phase of reflectarray antennas with high loss organic substrates
}

\author{
M. Y. Ismail' ${ }^{1}$, H. I Malik ${ }^{2}$, S. R. Masrol ${ }^{3}$, Sharmiza Adnan ${ }^{4}$ \\ 1,2,3 Universiti Tun Hussein Onn Malaysia (UTHM), Johor, Malaysia \\ ${ }^{4}$ Forest Research Institute Malaysia (FRIM), Selangor, Malaysia
}

\begin{abstract}
Article Info
Article history:

Received Oct 29, 2018

Revised Dec 15, 2018

Accepted Dec 26, 2018

\section{Keywords:}

Dielectric material

Paper substrate

Reflectarray antenna

Reflection phase
\end{abstract}

\begin{abstract}
This paper presents an analysis of reflection phase of reflectarray antenna based on high loss organic substrate material. Paper substrate material derived from recycled materials was characterized for dielectric material properties. The material shows excellent dielectric material properties with permittivity of 1.63 and loss tangent of 0.048 . Effect of substrate height variation over the reflection phase has been discussed. Full wave analysis of the simulated model depicts that the reduction in substrate height after a certain point results in reflection phase distortion of reflectarray element. Validation of the results has been done by simulation, fabrication and scattering parameters measurements of a rectangular patch element on proposed substrate material. The results show a broadband frequency response of $355 \mathrm{MHz}$ and a phase range of $301^{\circ}$ at a phase gradient of $0.14^{\circ} / \mathrm{MHz}$.
\end{abstract}

Copyright $@ 2019$ Institute of Advanced Engineering and Science. All rights reserved.

\section{Corresponding Author:}

Muhammad Yusof Ismail,

Faculty of Electrical and Electronic Engineering,

Universiti Tun Hussein Onn Malaysia,

Parit Raja, 86400, Batu Pahat, Johor Malaysia.

Email: yusofi@uthm.edu.my

\section{INTRODUCTION}

Microstrip reflectarray distinguish themselves from other reflector antenna due to their flat reflector profile. They have proven their capabilities for long range communication applications [1-3]. They combine the key features of phased array antennas and the dish reflectors. A typical microstrip reflectarray antenna consists of an array of radiating elements on a flat dielectric surface that is illuminated by a feed antenna. The elements on the array are illuminated in such a way to produce a collimating beam. Thus this mechanism eliminates the use of any microstrip feeding lines for the elements compared to phase arrays. This also cuts the losses in the array due to transmission lines, thus enhancing the antenna gain properties.

In order to maximize the antenna gain and directivity, proper phasing of individual elements on the array is required. This happens due to spatial phase difference caused by the flat surface of reflector and the incident spherical wavefront from the feed antenna. The incident wavefront has to cover different phase lengths in space to reach the reflectarray elements [4]. So at every element on the array phase compensation is done according to its position in the array. The phase uniformity across the array is achieve by incorporating different element shapes such as variable size patches, stub loaded delay lines and different types of slotted configuration of elements [5-9].

The reflection phase of the elements is desired to acquire a range of $360^{\circ}$ and it is an essential requirement of reflectarray design. An insufficient range may result in phase errors on the array thus resulting in the degraded radiation performance. Another important parameter for radiation performance of reflectarray is the reflection phase slope. A rapidly changing reflection phase results in high reflection phase sensitivity. Thus if the reflection phase sensitivity is higher than the etching tolerance then the phase errors cannot not be 
neglected and may result in performance degradation. The reflection phase range and the gradient of curve are directly related to each other, increasing the range will increase the phase sensitivity and vice versa. Another technique used for improving the phase range is using multi-layer configurations or dual resonance elements [10-14]. However the use of multiple layer structures pose design and fabrication challenges, while single layer dual band resonance configurations increase phasing complexity at both the resonances.

The performance of the reflectarray is highly dependent on the electrical properties of the substrate material. Substrate materials with lower dielectric permittivity are more desirable due to efficient radiation properties [15]. Advances in material technologies has brought in new and versatile materials to enhance the dielectric performances for reflectarray antennas. Innovative materials with latest manufacturing techniques are producing efficient reflectarray antenna designs for future communication systems [16, 17]. Besides using conventional material for reflectarray designs, researchers have reported different inorganic and organic materials such as using liquid crystals and textile based organic materials to acquire tunability and broadband frequency behavior [18-21].

In all the stated techniques and methods the reflection phase remains one of the key concerns in terms of its range and gradient to achieve admirable performances. This article discusses the introduction of a novel organic dielectric substrate material for reflectarray antenna to achieve broadband behavior. It discusses the effects of using high loss dielectric substrate materials on reflection phase of individual reflectarray elements. This articles presents the dielectric material characterization results of a proposed substrate, the simulation results along with a thorough discussion over the phase distortion behavior. In the end, measurement results of unit reflectarray elements embedded on proposed substrate are presented.

\section{ORGANIC DIELECTRIC SUBSTRATE MATERIAL}

\subsection{Material Composition}

The proposed dielectric substrate material was derived from recycles organic materials. The composition of the proposed paper substrate was carefully controlled to acquire suitable dielectric material properties. The paper is composed of two major constituents as stated below:

1. Recycled Catron Paper $(75 \%)$

2. Banana Pulp (25\%)

The recycled carton paper serves as the main component while the banana pulp acts as binding medium for the mixture particles due to its fibrous nature. The samples of the paper substrate were passed through different heating and drying stages in order to remove any moisture content that might affect the characterization results.

\subsection{Characterization Results}

The proposed dielectric materials were characterized for the electrical properties using a broadband material characterization techniques based on probe principle. The probe and the material under test are brought in tight contact and the probe measure the changes the in the fringing electromagnetic fields. A Speag $3.5 \mathrm{~mm}$ dielectric probe with an analysis range of $0.2-20 \mathrm{GHz}$ was used for characterization over the $\mathrm{X}$ - band. The results of material characterization are presented in Figure 1.

The results show the variation of the relative dielectric permittivity (cr) and the loss tangent ( $\tan \delta$ ) of the proposed material over the band of interest. Material characterization shows that the substrate provides a mean dielectric permittivity of 1.63 along with a dielectric loss tangent of 0.048 at $\mathrm{X}$ - band frequency operation.

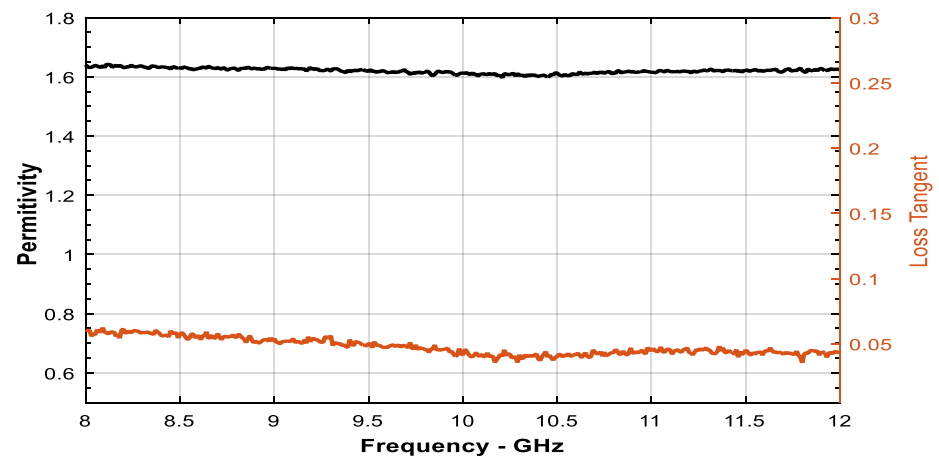

Figure 1. Dielectric material characterization results 


\section{EFFECT OF SUBSTRATE HEIGHT ON REFLECTION PHASE}

The proposed paper substrate material performance was analyzed by modelling a unit reflectarray element in a full-wave analysis tool. Using periodic conductive boundary conditions the unit element was simulated withTE10 mode of operation. The model under consideration is shown in Figure 2. The model consists of a rectangular patch element on a dielectric slab ground by a metallic ground plane. The element is placed with vertical boundaries are magnetic wall and horizontal boundaries are electric walls. The incident electric field vector is also shown in Figure 2. These conductive boundaries act as mirrors for the radiated electromagnetic energy and the element undergoes similar mutual coupling effects as it is being exciting in an infinite array of elements.

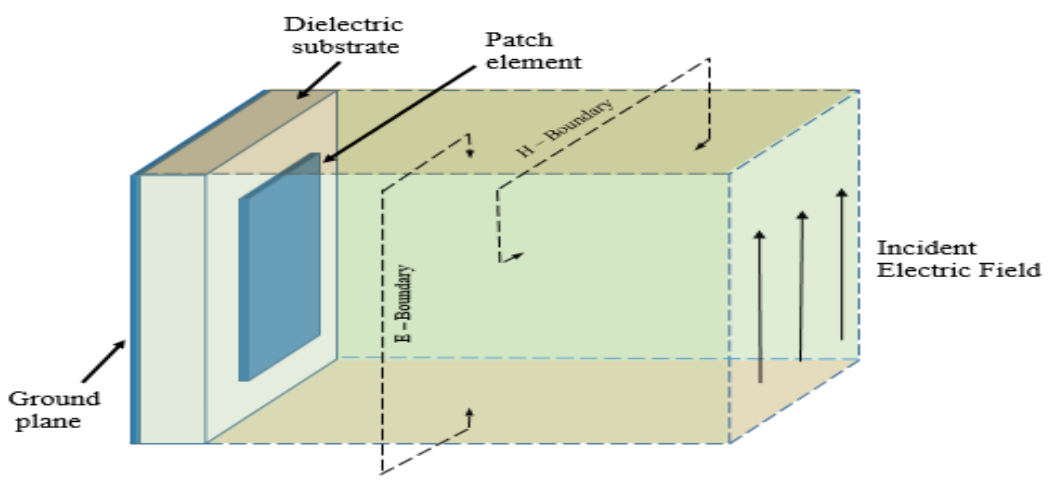

Figure 2. Full-wave simulation model of unit reflectarray element

In order to monitor the effect of substrate height over the reflection phase of the patch element the substrate height was swept for a range of $0.3-1.5 \mathrm{~mm}$. The simulation results for the reflection loss and the reflection phase are presented in Figure 3 and Figure 4 respectively. The reflection loss curves show that with the variation of substrate height from 0.15 to $1 \mathrm{~mm}$ the resonant frequency shifts from 11.58 to $10.54 \mathrm{GHz}$. Moreover it can be noticed from Figure 3 that with the increase in the substrate height the bandwidth of the element improves. The bandwidth is calculated by moving $10 \%$ above the maximum loss level.

An abnormality in the trend can be noticed in the Figure 3 that from 1-0.66 mm the reflection loss curves follow a typical trend of increased loss with reduced substrate height. However after 0.66 the loss starts to decrease again. This diversion from usual trend can also be noticed in the reflection phase curves where it can be seen that with the decrease in the substrate thickness from 1-0.66 mm the gradient of the reflection phase increases sharply from $0.23-0.59 \% \mathrm{MHz}$. After further decrease in the substrate height the phase curve show an abnormal behavior where they cannot be unwrapped to normal phase range. This might be happening due to increase in the dielectric loss too much that the reflection phase is no longer following the generic trend. Thus a limit is to be defined for the substrate height to achieve fruitful reflection phase and reflection loss results.

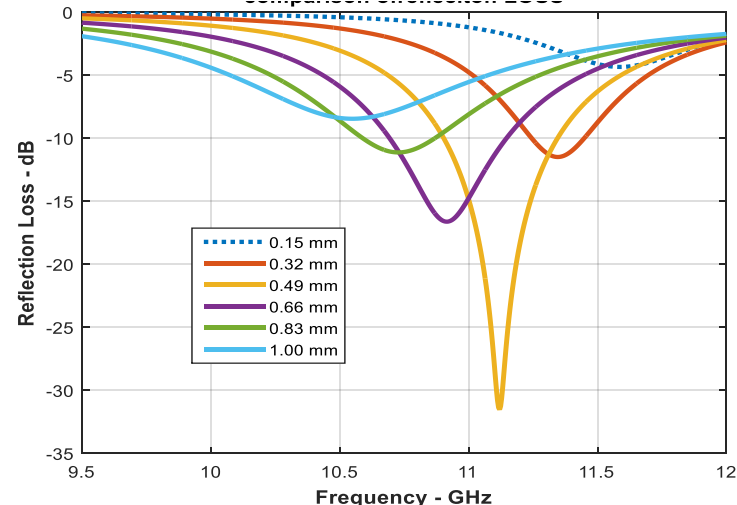

Figure 3. Reflection loss comparison for variable substrate heights

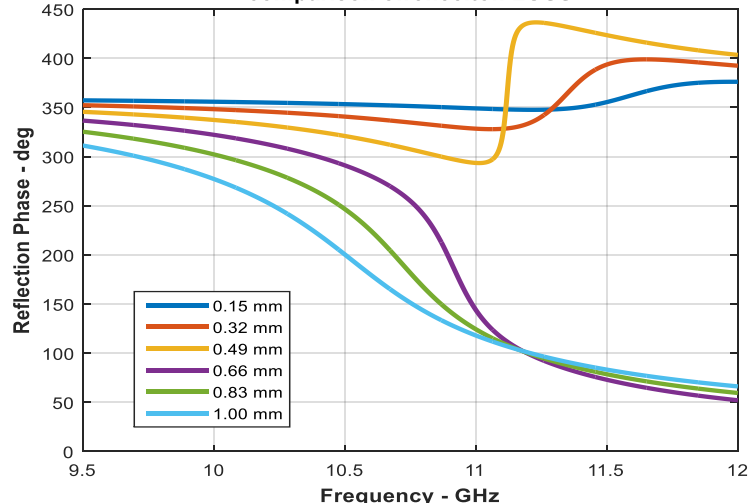

Figure 4. Reflection phase comparison for variable substrate heights 


\section{MEASUREMENTS AND RESULTS}

After the characterization of the organic substrate material and verification of the simulation design, validation of results was done by fabrication of unit reflectarray elements on proposed substrate. The fabrication was carried out using adhesive copper tape. Scattering parameter measurements were performance using an X-band tapered waveguide. The fabricated samples and the complete measurement setup are shown in Figure 5.

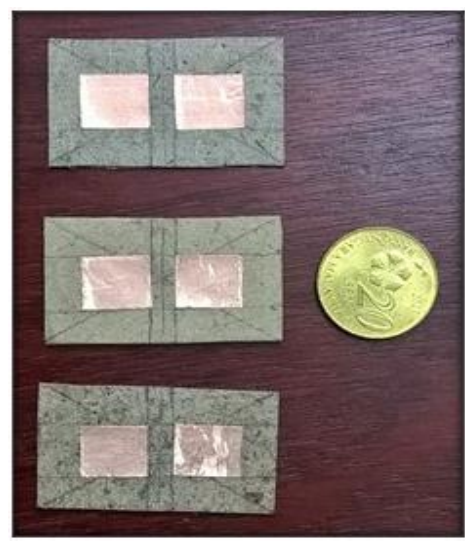

(a)

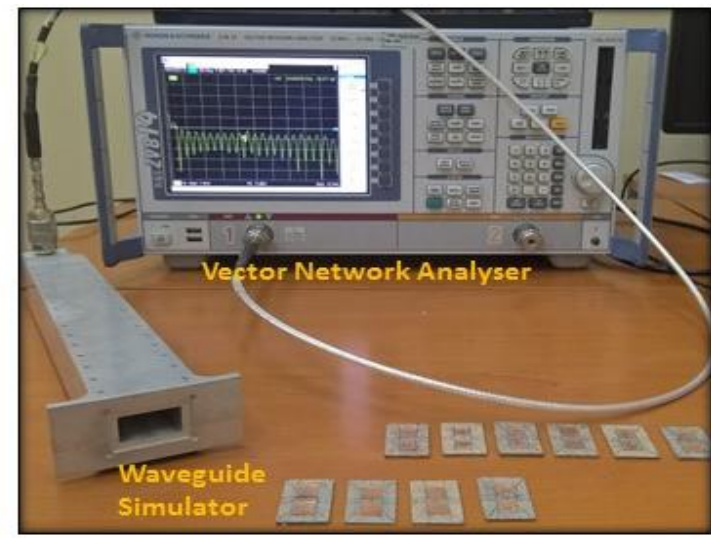

(b)

Figure 5. Fabrication and measurement (a) Fabricated sammples (b) Complete scattering paramters measurement setup

Figure 5(a) shows the fabricated samples on proposed substrate material. Multiple samples were fabricated and tested to achieve repeatability of results. Moreover the fabricated elements were measured for their fabrication tolerances and the differences in the dimension were incorporated in the simulation models. The setup presented in Figure 5(b) consists of a waveguide simulator attached to a Rodhe \& Schwarz $14 \mathrm{GHz}$ vector network analyzer via a coaxial cable. The scattering parameters were recorded and then a comparison was drawn between the simulated and the measured results.

The measured and simulated results of unit reflectarray elements on proposed organic substrate material are presented in Figure 6. The results presented in Figure 6 show a good agreement between the simulated and the measured results. The reflection loss curves show a resonance at $9.92 \mathrm{GHz}$ while the simulated model shows a resonance at $9.98 \mathrm{GHz}$. The simulated reflection loss curves show a maximum reflection loss of $-4.34 \mathrm{~dB}$ while the measured curve shows a loss of $-8.24 \mathrm{~dB}$. This increase in the reflection loss might be due to the measurement setup losses such as insertion loss and connector losses. The band width of the reflection loss curves is measured as $10 \%$ bandwidth. The results show bandwidths of 682 and $355 \mathrm{MHz}$ for simulated and measured reflection loss curves respectively.

The reflection phase curves presented in Figure 7 depict a close trend between the measured and the simulated curves. It can be noticed that the curves show maximum gradient at the resonance point of the element and the phase gradient decreases gradually before and after the resonance point. The reflection phase curves depict reflection phase gradients of 0.10 and $0.14 \% \mathrm{MHz}$ for simulated and measured phase curves. The phase curves cover phase ranges of $243^{\circ}$ and $301^{\circ}$ for simulated and measured results. It can be noticed that the measured reflection phase curves are greater than the simulated ranges. This occurs due to the increase in the loss of the reflectarray element since increase in loss results in a wider phase range.

The reflectarray unit elements above the proposed substrates show close agreement between the measured and the simulated results. The rippled present in the measured results of reflection loss curves are due to deviation of waveguide simulator from its ideal behavior. Moreover the presented results show that with low dielectric permittivity materials a broadband frequency response can be achieved. However this comes at a cost of reflection phase range and the gradient of the phase curve. Moreover while fabrication of the reflectarray antenna a minimum height of the substrate should be maintained to achieve accurate scattering parameter results. 


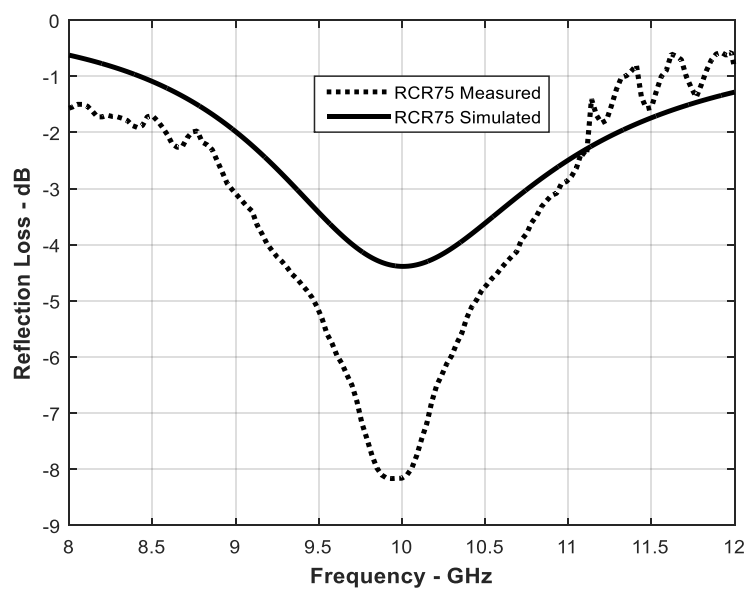

Figure 6. Reflection loss curves for measured and simulated results comparison

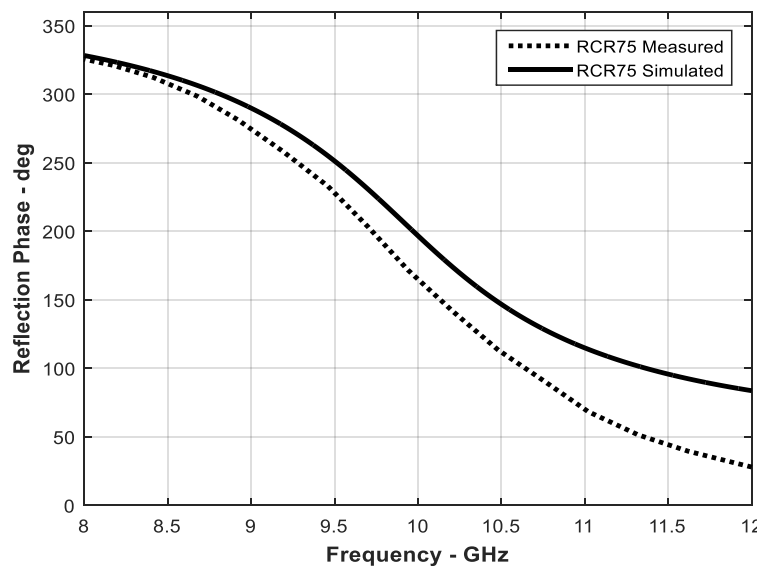

Figure 7. Comparison of measured and simulated reflection phase curves

\section{CONCLUSION}

Novel reflectarray antenna elements based on organic substrate material has been presented for $\mathrm{X}$ band frequency operation. The characterization of the material shows excellent dielectric properties for broadband frequency operation. The analysis of the effect of substrate height variation over the scattering parameters show that during the designing of the reflectarray unit elements a minimum substrate height must be maintained to acquire suitable reflection phase curves. Unit reflectarray elements fabricated over the proposed substrate material show broadband frequency behavior of $355 \mathrm{MHz}$ bandwidth, along with an adequate phase range coverage of $301^{\circ}$.

\section{ACKNOWLEDGEMENTS}

The work was funded by GPPS Grant (VOT466), RACE Grant (VOT1119) and Research Fund UTHM awarded by Ministry of Higher Education Malaysia.

\section{REFERENCES}

[1] J. Huang, "Capabilities of printed reflectarray antennas," in Proceedings of International Symposium on Phased Array Systems and Technology, pp. 131-134.

[2] J. Huang, "Spacecraft Antenna Research and Development Activities Aimed at Future Missions," in Spaceborne Antennas for Planetary Exploration, Hoboken, NJ, USA: John Wiley \& Sons, Inc., 2006, pp. 485-536.

[3] P. Nayeri, F. Yang, and A. Z. Elsherbeni, "Beam-scanning reflectarray antennas: A technical overview and state of the art," IEEE Antennas Propag. Mag., vol. 57, no. 4, pp. 32-47, Aug. 2015.

[4] J. Huang and J. A. Encinar, Reflectarray antennas. IEEE, 2007.

[5] C. Tienda, J. A. Encinar, M. Arrebola, M. Barba, and E. Carrasco, "Design, manufacturing and test of a dualreflectarray antenna with improved bandwidth and reduced cross-polarization," IEEE Trans. Antennas Propag., vol. 61, no. 3, pp. 1180-1190, Mar. 2013.

[6] R. S. Malfajani and Z. Atlasbaf, "Design and Implementation of a Dual-Band Single Layer Reflectarray in X and K Bands," IEEE Trans. Antennas Propag., vol. 62, no. 8, pp. 4425-4431, Aug. 2014.

[7] J. H. Yoon, Y. J. Yoon, W. Lee, and J. So, "Square Ring Element Reflectarrays With Improved Radiation Characteristics by Reducing Reflection Phase Sensitivity," IEEE Trans. Antennas Propag., vol. 63, no. 2, pp. 814818, Feb. 2015.

[8] X. Xia, Q. Wu, H. Wang, C. Yu, and W. Hong, "Wideband Millimeter-Wave Microstrip Reflectarray Using DualResonance Unit Cells,” IEEE Antennas Wirel. Propag. Lett., vol. 16, pp. 4-7, 2017.

[9] B. D. Nguyen, K. T. Pham, V. S. Tran, L. Mai, and N. Yonemoto, "Reflectarray element using cut-ring patch coupled to delay line," IEEE Antennas Wirel. Propag. Lett., vol. 14, pp. 571-574, 2015.

[10] J. A. Encinar and J. A. Zornoza, "Three-layer printed reflectarrays for contoured beam space applications," IEEE Trans. Antennas Propag., vol. 52, no. 5, pp. 1138-1148, May 2004.

[11] C. Han, J. Huang, and K. Chang, "A high efficiency offset-fed X/Ka-dual-band reflectarray using thin membranes," IEEE Trans. Antennas Propag., vol. 53, no. 9, pp. 2792-2798, Sep. 2005.

[12] S.-W. Qu, S. Member, Q. Chen, and M.-Y. Xia, "Single-Layer Dual-Band Re fl ectarray With Single Linear Polarization," 2012 Int. Conf. Microw. Millim. Wave Technol., vol. 62, no. 1, pp. 199-205, May 2014. 
[13] J. A. Encinar et al., "Dual-Polarization Reflectarray in Ku-band Based on Two Layers of Dipole-Arrays for a Transmit-Receive Satellite Antenna with South American Coverage," 2017 11th Eur. Conf. Antennas Propag., pp. 80-83, Mar. 2017.

[14] T. Cai, G.-M. Wang, and X.-F. Zhang, "Compact Dual-Resonance Element with Low Phase Sensitivity for Offset Reflectarray Antennas," IEEE Antennas Wirel. Propag. Lett., vol. 16, pp. 1213-1216, 2017.

[15] C. A. Balanis, "Antenna theory: analysis and design", 3rd ed. John Wiley \& Sons, Inc., 2005.

[16] P. Nayeri et al., "3D Printed Dielectric Reflectarrays: Low-Cost High-Gain Antennas at Sub-Millimeter Waves," IEEE Trans. Antennas Propag., vol. 62, no. 4, pp. 2000-2008, Apr. 2014.

[17] B.-J. Chen, H. Yi, K. B. Ng, S.-W. Qu, and C. H. Chan, "3D Printed Reflectarray Antenna at $60 \mathrm{GHz}$," $3 D$ Print. Reflectarray Antenna $60 \mathrm{GHz}$, pp. 92-93, 2016.

[18] W. Hu et al., "Liquid-crystal-based reflectarray antenna with electronically switchable monopulse patterns," Electron. Lett., vol. 43, no. 14, p. 744, 2007.

[19] M. H. Dahri and M. Y. Ismail, "Performance Analysis of Reflectarray Resonant Elements based on Dielectric Anisotropic Materials," Procedia Eng., vol. 53, pp. 203-207, Jan. 2013.

[20] M. M. Tahseen and A. A. Kishk, "C-Band flexible and portable circularly polarized textile-reflectarray (TRA)," in 2017 IEEE International Symposium on Antennas and Propagation \& USNC/URSI National Radio Science Meeting, 2017, pp. 87-88.

[21] M. M. Tahseen and A. A. Kishk, "Practical Investigation of Different Possible Texitile Unit Cell for a C-band Portable Textile Reflectarray Using Conductive Thread,” Prog. Electromagn. Res. B, vol. 66, pp. 15-29, 2016. 